\title{
Blind Reduced-Rank MMSE Detector for DS-CDMA Systems
}

\author{
Xiaodong Cai \\ Department of Electrical and Computer Engineering, University of Minnesota, Minneapolis, MN 55455, USA \\ Email: caixd@ece.umn.edu
}

\section{Hongya Ge}

Department of Electrical and Computer Engineering, New Jersey Institute of Technology, Newark, NJ 07102, USA Email: ge@adm.njit.edu

\author{
Ali N. Akansu \\ Department of Electrical and Computer Engineering, New Jersey Institute of Technology, Newark, NJ 07102, USA \\ Email:akansu@njit.edu
}

Received 18 March 2002 and in revised form 14 August 2002

\begin{abstract}
We first develop a reduced-rank minimum mean square error (MMSE) detector for direct-sequence (DS) code division multiple access (CDMA) by forcing the linear MMSE detector to lie in a signal subspace of a reduced dimension. While a reduced-rank MMSE detector has lower complexity, it cannot outperform the full-rank MMSE detector. We then concentrate on the blind reduced-rank MMSE detector which is obtained from an estimated covariance matrix. Our analysis and simulation results show that when the desired user's signal is in a low-dimensional subspace, there exists an optimal subspace so that the blind reducedrank MMSE detector lying in this subspace has the best performance. By properly choosing a subspace, we guarantee that the optimal blind reduced-rank MMSE detector is obtained. An adaptive blind reduced-rank MMSE detector, based on a subspace tracking algorithm, is developed. The adaptive blind reduced-rank MMSE detector exhibits superior steady-state performance and fast convergence speed.
\end{abstract}

Keywords and phrases: CDMA, blind multiuser detection, reduced-rank, MMSE, subspace tracking.

\section{INTRODUCTION}

The major limitation on the performance and channel capacity of direct-sequence (DS) code division multiple access (CDMA) system is the multiple-access interference (MAI) due to simultaneous transmissions. The conventional matched filter (MF) detector cannot suppress MAI effectively, and it suffers from the near-far problem. Since CDMA is not fundamentally MAI limited [1], multiuser detection (MUD) techniques can substantially improve the performance of a CDMA system. While the optimal multiuser detector, which is essentially a maximum-likelihood (ML) sequence detector, has prohibitive complexity, many other multiuser detectors with relatively low complexity such as decision feedback detector, successive or parallel interference canceler, and linear multiuser detectors have been developed [1]. The linear decorrelator removes all cross-correlations between active users and thereby eliminates MAI at the price of enhancing noise [2]. The linear minimum mean square error (MMSE) detector is the optimal linear detector that maximizes signal-to-interference ratio (SIR) [3, 4]. The blind MMSE detector proposed in [5] minimizes the receiver's mean output energy (MOE) while constraining the response of the desired user to a constant. It was shown that MOE and MMSE are directly related and minimizing one is equivalent to minimizing the other [5]. In [6], both blind decorrelator and blind MMSE detector were derived in terms of the signal space parameters; and an adaptive blind MMSE detector was developed, based on a subspace tracking algorithm [7]. It was shown that the blind adaptive MMSE detector obtained from the signal space parameters has much better steady-state performance compared with the blind MOE detector [6]. While blind MUD in [6] mainly focuses on Gaussian channel, the subspace approach to blind MUD in [6] has been extended to multipath channels $[8,9]$.

The blind MMSE detector proposed in $[6,8,9]$ lies in the whole signal space. In this paper, we first investigate the reduced-rank MMSE detector that lies in a subspace of the signal space under the assumption that the detector has all active users' information. In CDMA systems, 
active users typically transmit with different powers; thus, the desired user may lie in a subspace that has a lower dimension than the signal space. When this subspace is perfectly known by the detector, the received data can be projected onto this subspace to provide a sufficient statistic for detecting the desired user's bits. When the desired signal lies in the whole signal subspace, it may still be possible to choose a lower-dimensional subspace so that the performance loss is negligible. Note that MUD, based on the projected signal in a low-dimension subspace, has lower complexity and better convergence speed if adaptive MUD is considered.

Since the desired user may not have the knowledge of other users' spreading codes and powers, a blind multiuser detector is well motivated for a mobile user to suppress MAI. The blind MMSE detector in [6] is constructed from the signal space parameters which are computed from an estimated covariance matrix of the received signal. In this paper, based on the estimated covariance matrix, we study all possible blind MMSE detectors lying in different subspaces [10] and observe that there always exists an optimal subspace, in which the blind reduced-rank lies, achieves the highest SIR. Suppose that the dimension of the signal subspace is $K$. While the blind MOE detector in [5] is full rank, the blind MMSE detectors in $[6,8,9]$ are rank- $K$ MMSE detectors in the context of our general blind reduced-rank MMSE detectors with different ranks. It was observed that the blind rank$K$ MMSE detector outperforms the blind full-rank MOE detector [6]. By changing the rank and properly selecting a subspace, we can obtain the optimal blind reduced-rank MMSE detector that achieves the highest SIR. Since the blind rank- $K$ MMSE detector is only a special case of our blind reduced-rank MMSE detector, our optimal blind reducedrank MMSE detector achieves better or the same performance compared with the blind rank- $K$ MMSE detector. The reduced-rank MMSE detector for DS-CDMA, based on the multistage Wiener filter in [11], was analyzed in [12] under the assumption that the covariance matrix is known. Our reduced-rank MMSE detector uses the cross spectral metric (CSM) approach in [13] to select a subspace, and we focus on the blind MMSE detector which uses an estimated covariance matrix.

The rest of this paper is organized as follows. In Section 2, after presenting the signal model for DS-CDMA and briefly introducing the linear MMSE detector, we derive a reducedrank MMSE detector which lies in a specific subspace of the signal space. We also develop the method to choose the best subspace among the subspaces of the same dimension. Section 3 analyzes the performance of the blind reducedrank detector which is obtained from the estimated covariance matrix. An adaptive blind reduced-rank MMSE detector, based on a subspace tracking algorithm, is developed in Section 4. Section 5 presents analytical and simulation results to demonstrate the performance of blind reduced-rank MMSE detectors with different ranks. Comparing these results reveals the advantage of the optimal blind reduced-rank MMSE detector. Conclusions are drawn in Section 6.

\section{SIGNAL MODEL AND REDUCED-RANK MMSE DETECTOR}

In this section, we present the signal model for DS-CDMA. After describing the conventional MMSE detector, we derive our reduced-rank MMSE detector. While derivation in this section assumes that the detector has all active users' information, we will concentrate in the remaining of this paper on the blind MMSE detector which has only the desired user's information.

\subsection{Signal model}

We consider a DS-CDMA system with $K$ users simultaneously transmitting over an additive white Gaussian noise (AWGN) channel. The received baseband signal can be modeled as

$$
r(t)=\sum_{k=1}^{K} A_{k} \sum_{i=-\infty}^{\infty} b_{k}(i) s_{k}\left(t-i T-\tau_{k}\right)+n(t),
$$

where $A_{k}$ is the received signal amplitude of user $k, b_{k}(i)$ is the information-bearing bit of user $k$ in the $i$ th bit interval, $s_{k}(t)$ is the normalized spreading waveform whose support is over the bit interval $[0, T], \tau_{k}$ is the propagation delay with respect to the receiver, and $n(t)$ is zeromean AWGN with power spectral density $\sigma^{2}$. We assume that $\left\{b_{k}(i)\right\}$ is a set of independent equiprobable \pm 1 random variables.

The spreading waveform $s_{k}(t)$ is expressed as

$$
s_{k}(t)=\frac{1}{\sqrt{N}} \sum_{j=0}^{N-1} s_{k}[j] \psi\left(t-j T_{c}\right), \quad t \in[0, T],
$$

where $\left\{s_{k}[0], s_{k}[1], \ldots, s_{k}[N-1]\right\}$ is the signature sequence of \pm 1 's assigned to the $k$ th user, $\psi(t)$ is a normalized chip waveform of duration $T_{c}$, and $N=T / T_{c}$ is the processing gain. If the transmitted signal passes through a multipath channel, we have the same signal model as (1) by replacing $s_{k}(t)$ with the effective spreading waveform which is given by convolution of $s_{k}(t)$ with channel impulse response. If all users are synchronous, that is, $\tau_{1}=\tau_{2}=\cdots=\tau_{K}=0$, it is then sufficient to consider the received signal during one bit interval, and the received signal model becomes

$$
r(t)=\sum_{k=1}^{K} A_{k} b_{k} s_{k}(t)+n(t), \quad t \in[0, T] .
$$

Since, given the received signal in $n$ symbol intervals, an asynchronous system of $K$ users can be viewed as equivalent to a synchronous system with $(n+1) K-1$ users [3], above synchronous signal model is general enough to subsume asynchronous signal model. Hence, throughout this paper, we will restrict our attention to the synchronous signal model (3). It is worth pointing out here that the work in this paper can be extended to multipath channel by incorporating blind channel identification in $[8,9]$ or by using a minimum variance detector [14]. 
Passing the received signal $r(t)$ through the chip-matched filter $\psi(T-t)$ and sampling at chip rate, we obtain an $N$ vector within a bit interval

$$
\mathbf{r}=\sum_{k=1}^{K} A_{k} b_{k} \mathbf{s}_{k}+\mathbf{n},
$$

where $\mathbf{s}_{k}=(1 / \sqrt{N})\left[s_{k}[0], s_{k}[1], \ldots, s_{k}[N-1]\right]^{T}$ is the normalized spreading code of the $k$ th user, and $\mathbf{n}$ is a white Gaussian noise vector with zero-mean and covariance matrix $\sigma^{2} \mathbf{I}_{N}$ ( $\mathbf{I}_{N}$ denotes the $N \times N$ identity matrix). We can also write the received signal vector in a compact matrix form as

$$
\mathbf{r}=\mathbf{S A b}+\mathbf{n},
$$

where $\mathbf{S}=\left[\mathbf{s}_{1}, \mathbf{s}_{2}, \ldots, \mathbf{s}_{K}\right], \mathbf{A}=\operatorname{diag}\left(A_{1}, A_{2}, \ldots, A_{K}\right)$, and $\mathbf{b}=$ $\left[b_{1}, b_{2}, \ldots, b_{K}\right]^{T}$.

\subsection{MMSE detector}

Suppose that the desired user is user 1, then the linear MMSE detector $\mathbf{c}$ for detecting this user's information bit minimizes the mean square error MSE $=E\left\{\left(b_{1}-c^{T} \mathbf{r}\right)^{2}\right\}$ and can be found as [3]

$$
\mathbf{c}=\mathbf{C}^{-1} \mathbf{p}
$$

where $\mathbf{p}=E\left\{b_{1} \mathbf{r}\right\}=A_{1} \mathbf{s}_{1}$ is the cross-correlation between $b_{1}$ and $\mathbf{r}$, and the covariance matrix $\mathbf{C}$ is given by

$$
\mathbf{C}=E\left\{\mathbf{r r}^{T}\right\}=\mathbf{S A}^{2} \mathbf{S}^{T}+\sigma^{2} \mathbf{I}_{N} .
$$

The decision on $b_{1}$ is given by $\hat{b}_{1}=\operatorname{sign}\left(\mathbf{c}^{T} \mathbf{r}\right)$, where $\operatorname{sign}(x)=1$ if $x>0$, and $\operatorname{sign}(x)=-1$ if $x<0$. The covariance matrix $\mathbf{C}$ can also be expressed in terms of its eigenvalue decomposition

$$
\mathbf{C}=\mathbf{U} \boldsymbol{\Lambda} \mathbf{U}^{T}=\left[\begin{array}{ll}
\mathbf{U}_{s} & \mathbf{U}_{n}
\end{array}\right]\left[\begin{array}{ll}
\boldsymbol{\Lambda}_{s} & \\
& \boldsymbol{\Lambda}_{n}
\end{array}\right]\left[\begin{array}{l}
\mathbf{U}_{s}^{T} \\
\mathbf{U}_{n}^{T}
\end{array}\right],
$$

where $\mathbf{U}=\left[\begin{array}{ll}\mathbf{U}_{s} & \mathbf{U}_{n}\end{array}\right], \boldsymbol{\Lambda}=\operatorname{diag}\left(\boldsymbol{\Lambda}_{s}, \boldsymbol{\Lambda}_{n}\right) ; \boldsymbol{\Lambda}_{s}=\operatorname{diag}\left(\lambda_{1}, \ldots\right.$, $\lambda_{K}$ ) contains the $K$ largest eigenvalues of $\mathbf{C}$ in descending order, and $\mathbf{U}_{s}=\left[\begin{array}{lll}\mathbf{u}_{1} & \cdots & \mathbf{u}_{K}\end{array}\right]$ contains the corresponding orthonormal eigenvectors; $\boldsymbol{\Lambda}_{n}=\sigma^{2} \mathbf{I}_{N-K}$ and $\mathbf{U}_{n}$ contains the $N-K$ orthonormal eigenvectors with the eigenvalue $\sigma^{2}$. The range space of $\mathbf{U}_{s}$ is called signal space since it has the same range as $\mathbf{S}$. The range of $\mathbf{U}_{n}$ is called noise space.

Since $\mathbf{s}_{1}$ is orthogonal to the noise space, it is easily shown that the full-rank MMSE detector in (6) can also be expressed in terms of the signal space parameters

$$
\mathbf{c}=\mathbf{U}_{s} \boldsymbol{\Lambda}_{s}^{-1} \mathbf{U}_{s}^{T} \mathbf{p}
$$

and mean square error is written as

$$
\text { MSE }=1-\mathbf{p}^{T} \mathbf{U}_{\mathbf{s}} \boldsymbol{\Lambda}_{s}^{-1} \mathbf{U}_{s}^{T} \mathbf{p} .
$$

It is observed from (9) that the MMSE detector $\mathbf{c}$ is a vector lying in the signal space.

\subsection{Reduced-rank MMSE detector}

Now, suppose that we restrict our linear detector to lie in a subspace of the signal space. The following result gives the linear MMSE detector under such restriction.

Proposition 1. Suppose that the matrix $\mathbf{U}_{r}$ contains $r(r \leq K)$ columns of $\mathbf{U}_{s}$ and $\boldsymbol{\Lambda}_{r}$ consists of corresponding eigenvalues. The rank-r MMSE detector, that lies in the range space of $\mathbf{U}_{r}$, is given by

$$
\mathbf{c}_{r}=\mathbf{U}_{r} \boldsymbol{\Lambda}_{r}^{-1} \mathbf{U}_{r}^{T} \mathbf{p},
$$

and the mean square error is found as

$$
\mathrm{MSE}_{r}=1-\mathbf{p}^{T} \mathbf{U}_{r} \boldsymbol{\Lambda}_{r}^{-1} \mathbf{U}_{r}^{T} \mathbf{p} .
$$

Proof. Let $\mathbf{w}=\left[w_{1}, w_{2}, \ldots, w_{r}\right]^{T}$ and $\mathbf{c}_{r}=\mathbf{U}_{r} \mathbf{w}$, then the mean square error is calculated by

$$
\begin{aligned}
\operatorname{MSE}_{r} & =E\left\{\left(b_{1}-\mathbf{c}_{r}^{T} \mathbf{r}\right)^{2}\right\} \\
& =\mathbf{c}_{r}^{T} \mathbf{C} \mathbf{c}_{r}-2 \mathbf{c}_{r}^{T} \mathbf{p}+1 \\
& =\mathbf{w}^{T} \mathbf{U}_{r}^{T} \mathbf{C} \mathbf{U}_{r} \mathbf{w}-2 \mathbf{w}^{T} \mathbf{U}_{r}^{T} \mathbf{p}+1 .
\end{aligned}
$$

Derivative of $\mathrm{MSE}_{r}$ with respect to $\mathbf{w}$ is found as

$$
\frac{\partial\left(\mathrm{MSE}_{r}\right)}{\partial \mathbf{w}}=2 \mathbf{U}_{r}^{T} \mathbf{C} \mathbf{U}_{r} \mathbf{w}-2 \mathbf{U}_{r}^{T} \mathbf{p}
$$

Letting it be equal to the $\mathbf{0}$ vector, we obtain

$$
\mathbf{w}=\left(\mathbf{U}_{r}^{T} \mathbf{C} \mathbf{U}_{r}\right)^{-1} \mathbf{U}_{r} \mathbf{p}=\boldsymbol{\Lambda}_{r}^{-1} \mathbf{U}_{r} \mathbf{p} .
$$

Here, we use the fact that the eigenvectors are orthonormal. The rank- $r$ MMSE detector in (11) follows from (15). Substituting $\mathbf{w}$ in (15) into (13), we obtain the mean square error in (12).

The rank-r MMSE detector can also be obtained as follows. The projection matrix for the range space of $\mathbf{U}_{r}$ is $\mathbf{P}_{r}=\mathbf{U}_{r} \mathbf{U}_{r}^{T}$. The projection of the received signal to the range of $\mathbf{U}_{r}$ is $\mathbf{r}_{1}=\mathbf{P}_{r} \mathbf{r}$. Then, the linear MMSE detector based on $\mathbf{r}_{1}$ is $\mathbf{c}_{r_{1}}=\mathbf{C}_{1}^{-1} \mathbf{p}_{1}$, where $\mathbf{C}_{1}$ is the covariance matrix of $\mathbf{r}_{1}$ and $\mathbf{p}_{1}$ is the cross-correlation between $\mathbf{r}_{1}$ and $b_{1}$. It is easily shown that $\mathbf{c}_{r_{1}}$ is the same as $\mathbf{c}_{r}$ in (11).

Comparing (10) and (12) reveals that the mean square error of a reduced-rank detector is greater than or equal to that of the full-rank detector. Hence, a reduced-rank MMSE detector cannot outperform the full-rank MMSE detector when the covariance matrix is perfectly known. Typically, the received signal powers of different users are different, which is true especially in the downlink. Then, the desired user may lie in a subspace of the signal space. In this case, if we choose $\mathbf{U}_{r}$ so that the desired user is in the range of $\mathbf{U}_{r}$, the reduced-rank MMSE detector has the same performance as the full-rank MMSE detector. If the desired user lies in the whole signal space for a given rank, we can choose the best subspace using the CSM approach to subspace selection in the context of a generalized sidelobe canceler [13]. We define 
a quantity $Q_{i}$ as

$$
Q_{i}=\frac{\left\|\mathbf{s}_{1}^{T} \mathbf{u}_{i}\right\|^{2}}{\lambda_{i}}
$$

where $Q_{i}$ can be viewed as the normalized energy of user 1 projected onto the $i$ th eigenvector. Then, MSE in (10) can also be expressed as

$$
\mathrm{MSE}=1-A_{1}^{2} \sum_{i=1}^{K} Q_{i}
$$

It is apparent from (17) that the optimal rank- $r$ MMSE detector lies in the subspace spanned by the $r$ eigenvectors corresponding to the $r$ largest $Q_{i}[13]$.

In the above derivation of the reduced-rank MMSE detector, it is assumed that the covariance matrix is known. In practice, a user knows his own spreading code but does not have the knowledge of other users' codes. In this case, the covariance matrix is estimated from a limited number of data samples; and the full-rank or reduced-rank MMSE detector can be blindly implemented based on the estimated covariance matrix without knowing other users' spreading codes. In the rest of the paper, we will focus on the blind reduced-rank MMSE detector. Using numerical and simulation results in Section 5, we will show that there exists an optimal blind reduced-rank MMSE detector which achieves the best performance among all MMSE detectors with different ranks.

\section{BLIND REDUCED-RANK MMSE DETECTOR AND PERFORMANCE ANALYSIS}

In this section, we present the blind reduced-rank MMSE detector and analyze its steady-state performance in terms of the mean square error at the detector output.

\subsection{Blind reduced-rank MMSE detector}

When $M$ samples of data vector $\left\{\mathbf{r}_{m}\right\}_{m=1}^{M}$ are available, the covariance matrix can be estimated by

$$
\hat{\mathbf{C}}=\frac{1}{M} \sum_{m=1}^{M} \mathbf{r}_{m} \mathbf{r}_{m}^{T}
$$

We assume that $\left\{\mathbf{r}_{m}\right\}_{m=1}^{M}$ are independent identically distributed (i.i.d.), which is true when the system is in steadystate, that is, there are no users exit or enter the system. Let $\hat{\boldsymbol{\Lambda}}=\operatorname{diag}\left(\hat{\lambda}_{1}, \ldots, \hat{\lambda}_{N}\right)$ contain the eigenvalues of $\hat{\mathbf{C}}$ in descending order and $\hat{\mathbf{U}}=\left[\hat{\mathbf{u}}_{1}, \ldots, \hat{\mathbf{u}}_{N}\right]$ contain the corresponding eigenvectors. Based on $\hat{\Lambda}$, the dimension of the signal subspace can be determined using information-theoretic criterion such as the Akaike information criterion (AIC) [15]. Suppose that the dimension of the signal space is $K$, let $\hat{\Lambda}_{s}$ contain the $K$ largest eigenvalues, and $\hat{\mathbf{U}}_{s}$ contain the corresponding eigenvectors. Given $0<r \leq K$, a blind rank- $r$ MMSE detector is then found from (11) as

$$
\hat{\mathbf{c}}_{r}=\hat{\mathbf{U}}_{r} \hat{\boldsymbol{\Lambda}}_{r}^{-1} \hat{\mathbf{U}}_{r}^{T} \mathbf{p}
$$

where $\hat{\mathbf{U}}_{r}$ comprises $r$ columns of $\hat{\mathbf{U}}_{s}$ and $\hat{\boldsymbol{\Lambda}}_{r}$ consists of corresponding eigenvalues. If we calculate $\hat{Q}_{i}=\left\|\mathbf{s}_{1}^{T} \hat{\mathbf{u}}_{i}\right\|^{2} / \hat{\lambda}_{i}$, the optimal rank- $r$ MMSE detector can be constructed from $r$ eigenvectors corresponding to the $r$ largest $\hat{Q}_{i}$. Without confusing with an arbitrary rank- $r$ MMSE detector in (19), we denote the optimal rank- $r$ MMSE detector as $\hat{\mathbf{c}}_{r}$. To determine the optimal rank for the reduced-rank MMSE detector, we apply the decided bits to each of $\left\{\hat{\mathbf{c}}_{r}\right\}_{r=1}^{K}$ and estimate MSE at each detector's output. The optimal rank corresponds to $\hat{\mathbf{c}}_{r}$ with the smallest MSE. The details of determining the dimension of the signal space using AIC and the optimal reducedrank MMSE detector in decision-directed mode will be presented in the adaptive blind reduced-rank MMSE detector in Section 4.

Remark 1. When the covariance matrix is known as we discussed in Section 2, the nonblind reduced-rank MMSE detector may have performance loss compared to the full-rank MMSE detector. However, when blind MMSE detector is considered, the estimation error in covariance matrix causes estimation error in the signal space and corresponding eigenvalues. Hence, a blind reduced-rank MMSE detector, lying in a properly selected subspace, can outperform the blind fullrank MMSE detector which will be shown in the analysis results and simulations in Section 5.

Remark 2. The detector $\hat{\mathbf{c}}_{r}$ in (19) does not need to know the interfering user's information. If the signal amplitude $A_{1}$ of the desired user is unknown to the detector, we can employ $\hat{\overline{\mathbf{c}}}_{r}=\hat{\mathbf{U}}_{r} \hat{\boldsymbol{\Lambda}}_{r}^{-1} \hat{\mathbf{U}}_{r}^{T} \mathbf{s}_{1}$ as a blind detector, which has the same output SIR as $\hat{\mathbf{c}}_{r}$ and is applicable to any constant-modulus (CM) constellation. Based on the CM property, we can estimate the signal amplitude $A_{1}$ by $\hat{A}_{1}=\sum_{m=1}^{N_{A}}\left|\hat{\overline{\mathbf{c}}}_{r}^{T} \mathbf{r}_{m}\right| / N_{A}$, where $N_{A}$ is the number of data to estimate $A_{1}$. Using $\hat{A}_{1}$ and decided bits, we obtain an estimate of MSE as $\sum_{m=1}^{N_{\mathrm{MSE}}}\left(\hat{b}_{1}(m)-\right.$ $\left.\hat{A}_{1} \hat{\overline{\mathbf{c}}}_{r}^{T} \mathbf{r}_{m}\right)^{2} / N_{\text {MSE }}$ for each of $\left\{\hat{\overline{\mathbf{c}}}_{r}\right\}_{r=1}^{K}$, where $N_{\text {MSE }}$ is the number of symbols used to estimate MSE. The optimal reducedrank detector $\hat{\overline{\mathbf{c}}}_{r}$ is the one that has the smallest MSE. Hence, the adaptive algorithm developed in Section 4 is also applicable to $\hat{\overline{\mathbf{c}}}_{r}$.

Remark 3. It is worth pointing out an alternative method to choose a near-optimal rank for $\hat{\mathbf{c}}_{r}$ or $\hat{\overline{\mathbf{c}}}_{r}$ without using decided bits. Let $K_{1}$ be the number of nonzero entries in $\left\{Q_{i}\right\}_{i=1}^{K}$, which is the dimension of the subspace in which $\mathbf{s}_{1}$ lies. Based on $\left\{\hat{Q}_{i}\right\}_{i=1}^{N}$, we can estimate $K_{1}$ using AIC. If the estimate of $K_{1}$ is $\hat{K}_{1}$, the reduced-rank detector is then constructed from $\hat{K}_{1}$ eigenvectors corresponding to the $\hat{K}_{1}$ largest $\hat{Q}_{i}$. The simulation results in Section 5 show that the rank- $K_{1}$ MMSE detector is not necessarily optimal, but its performance is close to that of the optimal reduced-rank MMSE detector. The advantage of this method of choosing subspace for the reducedrank detector is that the detector is not required to use decided bits to determine the optimal rank and may avoid the problem of unreliable decision. 


\subsection{Performance analysis}

First, we briefly review the asymptotic statistics of the eigenvalues and eigenvectors of the sample covariance matrix. Let $\Delta \lambda_{i}=\hat{\lambda}_{i}-\lambda_{i}$ and $\Delta \mathbf{u}_{i}=\hat{\mathbf{u}}_{i}-\mathbf{u}_{i}$. If $\left\{\mathbf{r}_{m}\right\}_{m=1}^{M}$ are i.i.d. Gaussian and all elements of $\left\{\lambda_{i}\right\}_{i=1}^{K}$ are distinct, we then know from [16, page 340] that $\left\{\Delta \lambda_{i}, \Delta \mathbf{u}_{i}\right\}_{i=1}^{K}$ are asymptotically normal with zero-mean and $\left\{\Delta \lambda_{i}\right\}_{i=1}^{K}$ is asymptotically independent of $\left\{\Delta \mathbf{u}_{i}\right\}_{i=1}^{K}$, as $M \rightarrow \infty$. The elements of $\left\{\Delta \lambda_{i}\right\}_{i=1}^{K}$ are mutually independent, and the variance of $\Delta \lambda_{i}$ is $\sigma_{i}^{2}=2 \lambda_{i}^{2} / M+O\left(1 / M^{2}\right)$; the covariances of $\left\{\Delta \mathbf{u}_{i}\right\}_{i=1}^{K}$ are given by [16, page 340]

$$
\mathbf{E}_{i j}= \begin{cases}\frac{1}{M} \sum_{\substack{n=1 \\ n \neq i}}^{N} \frac{\lambda_{i} \lambda_{n}}{\left(\lambda_{i}-\lambda_{n}\right)^{2}} \mathbf{u}_{n} \mathbf{u}_{n}^{T}+O\left(\frac{1}{M^{2}}\right), & i=j, \\ -\frac{\lambda_{i} \lambda_{j}}{M\left(\lambda_{i}-\lambda_{j}\right)^{2}} \mathbf{u}_{j} \mathbf{u}_{i}^{T}+O\left(\frac{1}{M^{2}}\right), & i \neq j .\end{cases}
$$

Although our data vector $\mathbf{r}_{m}$ is not Gaussian, the above statistic of eigenvalues and eigenvectors of the covariance matrix holds true due to the following reason. The derivation of the statistic of $\left\{\Delta \lambda_{i}, \Delta \mathbf{u}_{i}\right\}_{i=1}^{K}$ relies on asymptotic normality of the sample covariance matrix [16, page 454]. It is shown that when data vector is i.i.d. Gaussian, the sample covariance matrix is asymptotically normal by central limit theorem [17]. Since the central limit theorem does not require the data vector to be Gaussian and $\left\{\mathbf{r}_{m}\right\}_{m=1}^{M}$ to be i.i.d in our case, we know that $\hat{\mathbf{C}}$ is asymptotically normal; and thus, the above statistic of $\left\{\Delta \lambda_{i}, \Delta \mathbf{u}_{i}\right\}_{i=1}^{K}$ also holds true for any i.i.d. data vector.

The blind rank- $r$ detector $\hat{\mathbf{c}}_{r}$ is a random vector depending on the estimated eigenvalues and eigenvectors, and the mean square error conditioned on $\hat{\mathbf{c}}_{r}$ is also a random variable. Our objective is to obtain the mean of the conditional mean square error which is a good measure of the MMSE detector's performance. The approach is based on the secondorder Taylor series expansion of the conditional mean square error.

The mean square error conditioned on $\hat{\mathbf{c}}_{r}$ is given by

$$
\widehat{\operatorname{MSE}}_{r}=1+\hat{\mathbf{c}}_{r}^{T} \mathbf{C} \hat{\mathbf{c}}_{r}-2 \hat{\mathbf{c}}_{r}^{T} \mathbf{p} .
$$

The second-order Taylor series expansion of $\widehat{\mathrm{MSE}}_{r}$ around the point $\mathrm{P}=\left\{\mathbf{u}_{i}, \lambda_{i}\right\}_{i=1}^{r}$ is given by

$$
\begin{aligned}
\widehat{\operatorname{MSE}}_{r}= & \mathrm{MSE}_{r}+\sum_{i=1}^{r} \Delta \mathbf{u}_{i}^{T} \mathbf{g}_{i}+\sum_{i=1}^{r} \Delta \lambda_{i} f_{i}+\frac{1}{2} \sum_{i=1}^{r} \sum_{j=1}^{r} \Delta \lambda_{i} \Delta \mathbf{u}_{i}^{T} \mathbf{h}_{i j} \\
& +\frac{1}{2} \sum_{i=1}^{r} \sum_{j=1}^{r} \Delta \lambda_{i} \Delta \lambda_{j} F_{i j}+\frac{1}{2} \sum_{i=1}^{r} \sum_{j=1}^{r} \Delta \mathbf{u}_{i}^{T} \mathbf{H}_{i j} \Delta \mathbf{u}_{j},
\end{aligned}
$$

where the vector $\mathbf{g}_{i}$ is the gradient of $\widehat{\mathrm{MSE}}_{r}$ along $\hat{\mathbf{u}}_{i}$ at point $\mathrm{P}, f_{i}$ is the first partial derivative of $\widehat{\mathrm{MSE}}_{r}$ with respect to $\hat{\lambda}_{i}$ at point $\mathrm{P}, F_{i j}$ is the second partial derivative of $\widehat{\mathrm{MSE}}_{r}$ with respect to $\hat{\lambda}_{i}$ and $\hat{\lambda}_{j}$ at point $\mathrm{P}$, and the elements of vector $\mathbf{h}_{i j}$ and matrix $\mathbf{H}_{i j}$ are given by

$$
\left[\mathbf{h}_{i j}\right]_{m}=\left.\frac{\partial^{2} \widehat{\mathrm{MSE}}_{r}}{\partial \hat{\lambda}_{i} \partial \hat{\mathbf{u}}_{j}(m)}\right|_{P}, \quad\left[\mathbf{H}_{i j}\right]_{m n}=\left.\frac{\partial^{2} \widehat{\mathrm{MSE}}_{r}}{\partial \hat{\mathbf{u}}_{i}(m) \partial \hat{\mathbf{u}}_{j}(n)}\right|_{P} .
$$

Taking expectation of $\widehat{\mathrm{MSE}}_{r}$ with respect to $\left\{\Delta \lambda_{i}, \Delta \mathbf{u}_{i}, i=\right.$ $1, \ldots, r\}$, we obtain

$$
\widetilde{\mathrm{MSE}}_{r}=\mathrm{MSE}_{r}+\frac{1}{2} \sum_{i=1}^{r} \sigma_{i}^{2} F_{i i}+\frac{1}{2} \sum_{i=1}^{r} \sum_{j=1}^{r} \operatorname{tr}\left(\mathbf{H}_{i j} \mathbf{E}_{j i}\right),
$$

where $\operatorname{tr}(\cdot)$ denotes trace of the matrix in parentheses. Here, we use the fact that means of $\Delta \lambda_{i}$ and $\Delta \mathbf{u}_{i}$ are zero, elements of $\left\{\Delta \lambda_{i}\right\}_{i=1}^{r}$ are mutually independent, and $\left\{\Delta \lambda_{i}\right\}_{i=1}^{r}$ are independent of $\left\{\Delta \mathbf{u}_{i}\right\}_{i=1}^{r}$. Derivation of $\mathbf{H}_{i j}$ and $F_{i i}$ is given the appendix. In Section 5, we will evaluate MSE in (24) numerically. The numerical results based on the analysis in this section, along with simulations, will clearly show the advantage of the optimal blind reduced-rank MMSE detector.

\section{ADAPTIVE REDUCED-RANK MMSE DETECTOR BASED ON SUBSPACE TRACKING}

To implement the blind reduced-rank MMSE detector, we need to compute the eigenvectors and eigenvalues of the sample covariance matrix. This can be achieved by performing singular value decomposition (SVD) or eigenvalue decomposition (EVD) on the sample covariance matrix. Classical batch EVD and batch SVD algorithms [18] are computationally demanding. In order to overcome this difficulty, a number of adaptive algorithms for subspace tracking have been developed $[7,19,20]$. In [6], the projection approximation subspace tracking (PASTd) algorithm in [7] was used for blind adaptive multiuser detection. While PASTd algorithm has low-computational complexity $(O(N K))$, its convergence speed is very slow. In a CDMA system, users enter or leave the system randomly, and thus, the signal space changes dynamically. Hence, we need a fast convergence algorithm to track variations of the signal space. Furthermore, to find the optimal reduced-rank MMSE detector, orthogonal eigenvectors are required. However, the basis for the signal space tracked by PASTd are not orthogonal. For these reasons, instead of using PASTd, we use the subspace tracking algorithm in the reduced-rank adaptive filter named LORAF 1 in [20], which has a fast speed to track orthogonal eigenvectors. The subspace tracking algorithm in LORAF 1 has computational complexity of $O\left(N K^{2}\right)$. Since the subspace tracking algorithm in LORAF 1 cannot track the dimension of the signal space, we combine the rank tracking method in [21] with this algorithm. After briefly reviewing the adaptive subspace tracking algorithm in LORAF 1, we will incorporate rank tracking into this algorithm and develop our adaptive reduced-rank MMSE detector.

Suppose that we know the dimension of the signal space $K$. Let $\mathbf{U}_{0}$ be an $N \times K$ matrix with orthonormal columns, and denote the covariance matrix at time $t$ as $\mathbf{C}_{t}$. The following orthogonal iteration generates a sequence of 
$\operatorname{matrix}\left\{\mathbf{U}_{t}\right\}[18]$

$$
\begin{aligned}
\mathbf{A}_{t} & =\mathbf{C}_{t} \mathbf{U}_{t-1}, \\
\mathbf{A}_{t} & =\mathbf{U}_{t} \mathbf{R}_{t},
\end{aligned}
$$

where $\mathbf{U}_{t}$ and $\mathbf{R}_{t}$ are the factors of a skinny QR decomposition of the matrix $\mathbf{A}_{t}$. Assuming that $\mathbf{C}_{t}$ does not change in time, we can show that the sequence of the triangular matrix $\left\{\mathbf{R}_{t}\right\}$ will converge towards the diagonal matrix of $K$ dominant eigenvalues, and the sequence of recursion matrix $\left\{\mathbf{U}_{t}\right\}$ will converge towards the matrix of $K$ corresponding eigenvectors [18]. In our case, $\mathbf{C}_{t}$ is defined by $\mathbf{C}_{t}=\sum_{i=1}^{t} \beta^{t-i} \mathbf{r}_{i} \mathbf{r}_{i}^{T}$, where $0<\beta \leq 1$ and $\mathbf{C}_{t}$ is calculated recursively according to $\mathbf{C}_{t}=\beta \mathbf{C}_{t-1}+\mathbf{r}_{t} \mathbf{r}_{t}^{T}$. When $\mathbf{C}_{t}$ is updated at time $t$, we can calculate $\mathbf{U}_{t}$ and $\mathbf{R}_{t}$ using (25) and (26). A key step towards the fast subspace tracking algorithm is the orthogonal projection of the actual recursion matrix $\mathbf{U}_{t}$ onto the previous subspace spanned by the columns of $\mathbf{U}_{t-1}$,

$$
\mathbf{U}_{t}=\mathbf{U}_{t-1} \Theta_{t}+\Delta_{t}
$$

where $\boldsymbol{\Theta}_{t}=\mathbf{U}_{t-1}^{T} \mathbf{U}_{t}$ and $\Delta_{t}$ is orthogonal to the column space of $\mathbf{U}_{t-1}$. The update equation (25) becomes

$$
\mathbf{A}_{t}=\beta \mathbf{A}_{t-1} \Theta_{t-1}+\mathbf{r}_{t} \mathbf{z}_{t}^{T}+\beta \mathbf{C}_{t-1} \Delta_{t-1},
$$

where $\mathbf{z}_{t}^{T}=\mathbf{U}_{t-1}^{T} \mathbf{r}_{t}$. Only the last term requires $O\left(N^{2} K\right)$ operations, but it can be neglected without any performance penalty [20]. This results in the $O\left(N K^{2}\right)$ recursion for a direct updating of matrix $\mathbf{A}_{t}$,

$$
\mathbf{A}_{t}=\beta \mathbf{A}_{t-1} \Theta_{t-1}+\mathbf{r}_{t} \mathbf{z}_{t}^{T} .
$$

The fast subspace tracking algorithm consists of (26) and (29), where the diagonal elements of $\mathbf{R}_{t}$ comprise $K$ dominant eigenvalues and $\mathbf{U}_{t}$ contains corresponding eigenvectors.

Now we modify this algorithm to track both rank and the signal space as follows. Suppose that the rank at time $t-1$ is $K_{t-1}$, we track $K_{t-1}+1$ dominant eigenvalues $\left\{\lambda_{i}^{t}\right\}_{i=1}^{K_{t-1}+1}$ and corresponding eigenvectors in $\mathbf{U}_{t}$ at time $t$. Projecting received signal onto the orthogonal complement space to the span of $\mathbf{U}_{t}$, we obtain $\mathbf{x}=\left(\mathbf{I}-\mathbf{U}_{t} \mathbf{U}_{t}^{T}\right) \mathbf{r}_{t}$. From $\mathbf{x}$, we recursively calculate $\sigma_{t}^{2}=\beta \sigma_{t-1}^{2}+\mathbf{x}^{T} \mathbf{x} /\left(N-K_{t-1}-1\right)$ and let $\lambda_{i}^{t}=\sigma_{t}^{2}$ for $i=K_{t-1}+2, \ldots, N$. Based on the estimated eigenvalues $\left\{\lambda_{i}^{t}\right\}_{i=1}^{N}$, we can estimate the rank of the signal space using the AIC [15]. The quantity AIC is defined as

$$
\operatorname{AIC}(k)=(N-k) L \ln (\alpha(k))+k(2 N-k),
$$

where $L=1 /(1-\beta)$ and $\alpha(k)$ is given by

$$
\alpha(k)=\frac{\left(\sum_{i=k+1}^{N} \hat{\lambda}_{i}^{t}\right) /(N-k)}{\left(\prod_{i=k+1}^{N} \hat{\lambda}_{i}^{t}\right)^{1 /(N-k)}} .
$$

The estimated rank is given by $K_{t}=\arg \min _{0 \leq k \leq N-1} \operatorname{AIC}(k)+$ 1. If $K_{t}<K_{t-1}$, we remove the last columns from matrix $\mathbf{U}_{t}$ and update $K_{t}=K_{t-1}-1$. If $K_{t}>K_{t-1}$, we add $\mathbf{x} / \sigma_{t}^{2}$ to matrix $\mathbf{U}_{t}$ as its last column and let $K_{t}=K_{t-1}+1$.

After the eigenvectors and corresponding eigenvalues are obtained, we can find the optimal reduced-rank MMSE detector. The estimate of $Q_{i}$ in (16) is given by $\hat{Q}_{i}=\left\|\mathbf{s}_{1}^{T} \mathbf{u}_{i}^{t}\right\|^{2} / \lambda_{i}^{t}$. For every rank $0<r \leq K_{t}$, the optimal rank- $r$ MMSE detector $\hat{\mathbf{c}}_{r}$ is obtained, using the $r$ eigenvectors and eigenvalues corresponding to the $r$ largest $\hat{Q}_{i}$. Applying the recently decided bits to each of $K_{t}$ reduced-rank detectors $\left\{\hat{\mathbf{c}}_{r}, r=\right.$ $\left.1, \ldots, K_{t}\right\}$, we can estimate the mean square error at the output of each reduced-rank detector. The optimal reducedrank MMSE detector is the one having the smallest mean square error. Mathematically, if $\operatorname{MSE}(r)$ is the MSE at the output of $\hat{\mathbf{c}}_{r}$, the optimal reduced-rank MMSE detector is given by

$$
\mathbf{c}_{o}=\arg \min _{\left\{\hat{\mathbf{c}}_{r}\right\}_{r=1}^{K_{t}}} \operatorname{MSE}(r) .
$$

The proposed adaptive reduced-rank MMSE detector is summarized in Table 1, in which forgetting factor is chosen to be $\beta=0.995$, and $N_{\mathrm{MSE}}=10$ recently decided bits are used to estimate MSE. In the initializing stage, choosing a rank less than the dimension of the signal space may not be reliable because MSE cannot be estimated accurately. So, the rank- $K_{t}$ MMSE detector should be used in this stage. After that, since the optimal reduced-rank MMSE detector is chosen from all MMSE detectors with different ranks, it has the best possible steady-state performance and the fastest convergence speed if the signal space is disturbed when a user exits or enters the system. This is confirmed by the simulation results in Section 5 .

\section{SIMULATION AND ANALYSIS RESULTS}

In this section, we provide some simulation examples and numerical results based on the analysis in Section 3 to demonstrate the performance of the reduced-rank MMSE detector. For a given rank $r \leq K$, the optimal blind rank- $r$ MMSE detector is chosen to lie in the subspace spanned by $r$ eigenvectors corresponding to the $r$ largest $\hat{Q}_{i}$ as discussed in Section 3. In simulations, we also show the performance of the MMSE detectors constructed from $r>K$ eigenvectors of the sample covariance matrix, where $r-K$ eigenvectors are arbitrarily chosen from $N-K$ eigenvectors spanning the noise space. The performance measure for simulation is SIR. Since the distribution of a linear MMSE detector's output is approximately Gaussian [22], the output SIR translates easily into an equivalent bit error probability. In analysis results, the performance measure is mean square error which is given by (24). Gold sequences are used as spreading codes. Unless otherwise stated, the processing gain is $N=31$ and the data plotted are averaged over 400 independent runs. The number of data samples used to estimate covariance matrix is denoted as $M$. User 1 is assumed to be the desired user; SNR is defined as $\mathrm{SNR}=A_{1}^{2} / \sigma^{2}$ and is chosen as $\mathrm{SNR}=20 \mathrm{~dB}$ in all plots.

Example 1 (simulation of a single user system). The optimal detector in the single user case is the MF detector. However, 
Initialization $\mathbf{U}_{0}=\mathbf{I}_{N} ; \boldsymbol{\Theta}_{0}=\mathbf{I}_{N} ; \mathbf{A}=\mathbf{0}_{N} ; K_{0}=N-1 ; \beta=0.995 ;$ $\sigma_{0}^{2}=0 ; N_{\mathrm{MSE}}=10$

Update $K_{t-1}+1$ eigenvectors in $\mathbf{U}_{t}$ and $N$ eigenvalues $\left\{\lambda_{i}^{t}\right\}_{i=1}^{N}$

$\mathbf{z}_{t}=\mathbf{U}_{t-1}^{T} \mathbf{r}_{t}$

$\mathbf{A}_{t}=\beta \mathbf{A}_{t-1} \boldsymbol{\Theta}_{t-1}+\mathbf{r}_{t} \mathbf{z}_{t}^{T}$

$\mathbf{A}_{t}=\mathbf{U}_{t} \mathbf{R}_{t}:$ QR factorization

$\boldsymbol{\Theta}_{t}=\mathbf{U}_{t-1}^{T} \mathbf{U}_{t}$

$\mathbf{x}=\mathbf{r}_{t}-\mathbf{U}_{t} \mathbf{U}_{t}^{T} \mathbf{r}_{t}$

$\sigma_{t}^{2}=\beta \sigma_{t-1}^{2}+\mathbf{x}^{T} \mathbf{x} /\left(N-K_{t-1}-1\right)$

$\left\{\lambda_{k}^{t}=\mathbf{R}_{t}(k, k)\right\}_{k=1}^{K_{t-1}+1},\left\{\lambda_{k}^{t}=\sigma_{t}^{2}\right\}_{k=K_{t-1}+2}^{N}$

Update the rank of signal subspace $K_{t}$

FOR $k=1: N-1$ DO

$$
\begin{aligned}
& \alpha(k)=\left[\sum_{i=k+1}^{N} \lambda_{i}^{t} /(N-k)\right] /\left(\prod_{i=k+1}^{N} \lambda_{i}^{t}\right)^{1 /(N-k)} \\
& \operatorname{AIC}(k)=(N-k) \ln [\alpha(k)] /(1-\beta)+k(2 N-k)
\end{aligned}
$$

END

$$
K_{t}=\arg \min _{0 \leq k \leq N-1} \operatorname{AIC}(k)+1
$$

IF $K_{t}<K_{t-1}$, then

$$
\begin{aligned}
& K_{t}=K_{t-1}-1 \\
& \mathbf{U}_{t}=\mathbf{U}_{t}\left(:, 1: K_{t-1}\right) \\
& \mathbf{A}_{t}=\mathbf{A}_{t}\left(:, 1: K_{t-1}\right) \\
& \boldsymbol{\Theta}_{t}=\boldsymbol{\Theta}_{t}\left(1: K_{t-1}, 1: K_{t-1}\right)
\end{aligned}
$$

ELSE IF $K_{t}>K_{t-1}$ THEN

$$
\begin{aligned}
& K_{t}=K_{t-1}+1 \\
& \mathbf{U}_{t}=\left[\mathbf{U}_{t}, \mathbf{x} / \sigma_{t}^{2}\right] \\
& \mathbf{A}_{t}=\left[\mathbf{A}_{t}, \mathbf{x}\right] \\
& \theta_{t}=\left[\frac{\theta_{t}}{\mathbf{0}} \frac{\mathbf{0}}{1}\right]
\end{aligned}
$$

END

Choose the optimal reduced-rank MMSE detector $\mathbf{c}_{o}$

calculate $\left\{\hat{Q}_{i}\right\}_{i=1}^{K_{t}}$, and generate a matrix $\mathbf{V}$ whose first column $\mathbf{v}_{1}$ corresponds to the largest $\left\{Q_{i}\right\}_{i=1}^{K_{t}}$ and second column $\mathbf{v}_{2}$ corresponds to the second largest $\left\{Q_{i}\right\}_{i=1}^{K_{t-1}}$, and so on. Let $\eta_{i}$ be the eigenvalue corresponding to $\mathbf{v}_{i}$

$\hat{\mathbf{c}}_{0}=\mathbf{0}$

FOR $r=1: K_{t} \mathrm{DO}$

$$
\begin{aligned}
& \hat{\mathbf{c}}_{r}=\hat{\mathbf{c}}_{r-1}+\mathbf{v}_{k} \mathbf{v}_{k}^{T} \mathbf{p} / \eta_{k} \\
& \operatorname{MSE}(r)=0 \\
& \operatorname{FOR} i=1: N_{\mathrm{MSE}} \mathrm{DO} \\
& \quad \operatorname{MSE}(r)=\operatorname{MSE}(r)+\left(\hat{b}_{t-i}-\hat{\mathbf{c}}_{r}^{T} \mathbf{r}_{t-i}\right)^{2}
\end{aligned}
$$

END

END

$$
\begin{aligned}
& \mathbf{c}_{o}=\arg \min _{\left\{\hat{\mathbf{c}}_{r}\right\}_{r=1}^{K_{t}}} \operatorname{MSE}(r) \\
& \hat{b}_{t}=\operatorname{sign}\left(\mathbf{c}_{o}^{T} \mathbf{r}_{t}\right)
\end{aligned}
$$

Algorithm 1: The adaptive algorithm for the reduced-rank MMSE detector.

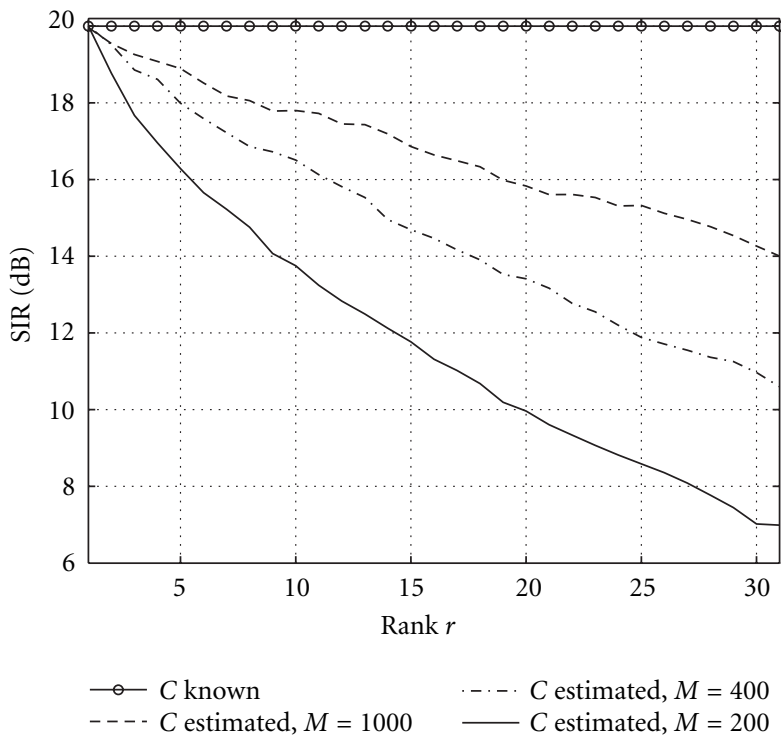

FIgURE 1: SIR of MMSE detectors. $N=31, K=1, \mathrm{SNR}=20 \mathrm{~dB}$.

an MMSE detector designed for suppressing MAI is likely to operate in a single user environment because a user does not have other active users' information. The simulated performance of a single user system is plotted in Figure 1. Since the noise-free signal has rank 1 , the performance of all detectors with different ranks is the same when the covariance matrix is known. When the covariance matrix is estimated, we see that the optimal blind reduced-rank MMSE has rank 1. If we use more eigenvectors other than the one corresponding to the largest $\hat{Q}_{i}$ to construct the MMSE detector, we get more noise than the desired signal at the output of the detector, which degrades the performance. We also observed from Figure 1 that the number of samples $M$ used to estimate the covariance matrix affects the performance of rank- $r(r>1)$ detectors. For example, SIR at the full-rank MMSE detector output is $7 \mathrm{~dB}$ for $M=200$, and $14 \mathrm{~dB}$ for $M=1000$.

Example 2 (simulation of 10 user systems). In Figure 2, nine interference users' SNRs are $10 \mathrm{~dB}$. This may be the case in which there is only one user in the cell but there exists inter-cell interference. From the SIR curve of the MMSE detector with known covariance matrix, we see that the signature of the desired user lies in a two-dimensional subspace, and most of the desired user's energy is in a onedimensional subspace. Hence, a rank-1 blind MMSE detector has the best performance. In Figure 3, there are five weak interfering users and four strong interfering users. The optimal blind reduced-rank MMSE detector has rank 2. Figure 4 is for the perfect power control case, where nine interfering users have the same power as the desired user. In this case, rank-K MMSE detector has the best performance since the signature of the desired user is in the whole signal space. 


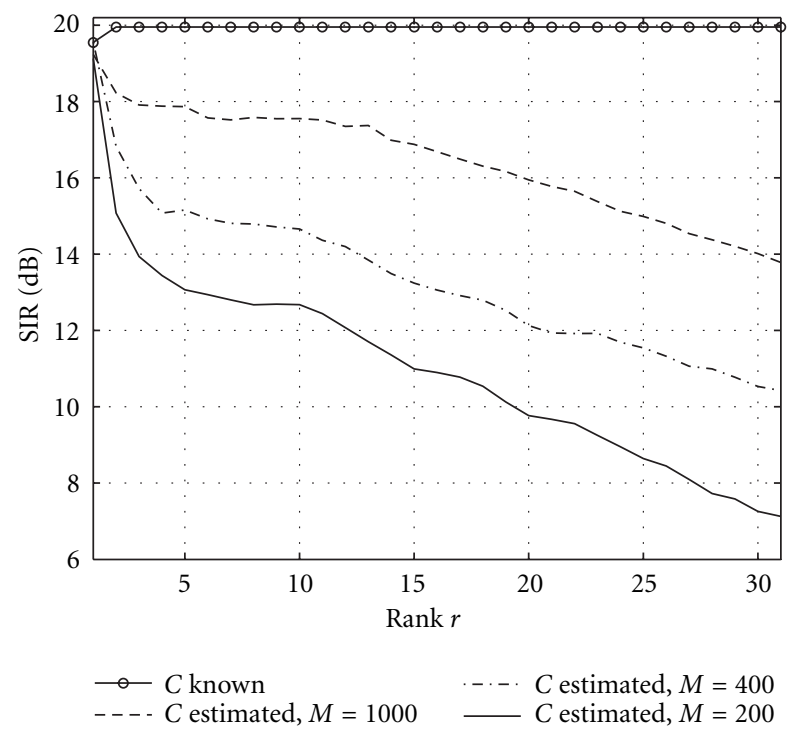

FIGURE 2: SIR of MMSE detectors. $N=31, K=10$, SNR $=20 \mathrm{~dB}$, $(K-1)$ interferences' SNRs $10 \mathrm{~dB}$.

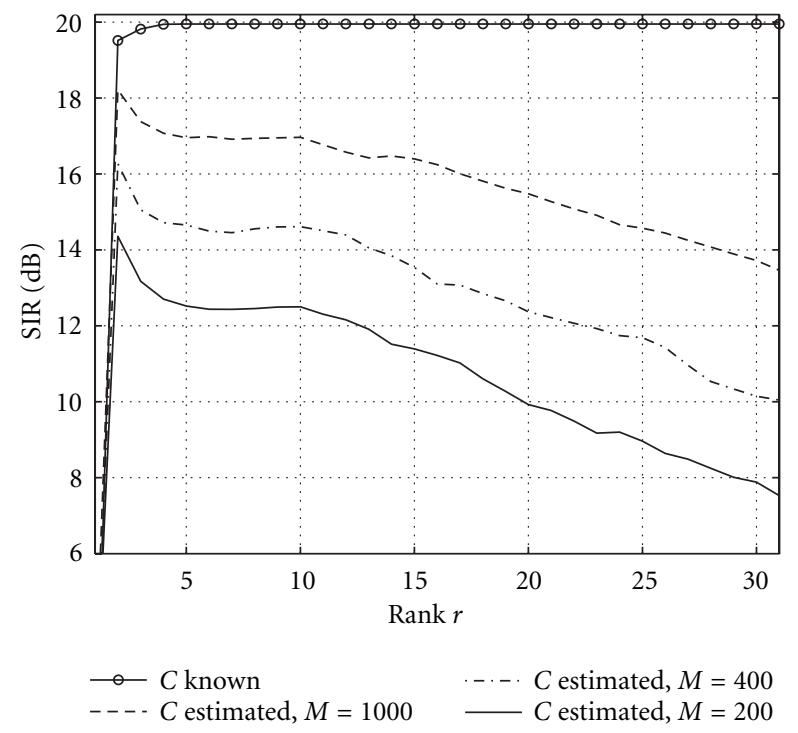

FIgURE 3: SIR of MMSE detectors. $N=31, K=10$, SNR $=20 \mathrm{~dB}$, $(K-1)$ interferences' SNRs are $10 \mathrm{~dB}, 10 \mathrm{~dB}, 10 \mathrm{~dB}, 10 \mathrm{~dB}, 10 \mathrm{~dB}$, $20 \mathrm{~dB}, 30 \mathrm{~dB}, 30 \mathrm{~dB}, 40 \mathrm{~dB}$.

All simulations show that the MMSE detectors whose rank are greater than $K$ perform worse than the rank- $K$ MMSE detector. Figure 5 compares the performance of the optimal reduced-rank MMSE detector, the rank- $K$ MMSE detector, and the full-rank MMSE detector when the number of samples used to estimate the covariance matrix changes. The users' powers are the same as in Figure 3. We observe that the optimal reduced-rank MMSE detector needs about half number of samples to achieve the same performance as

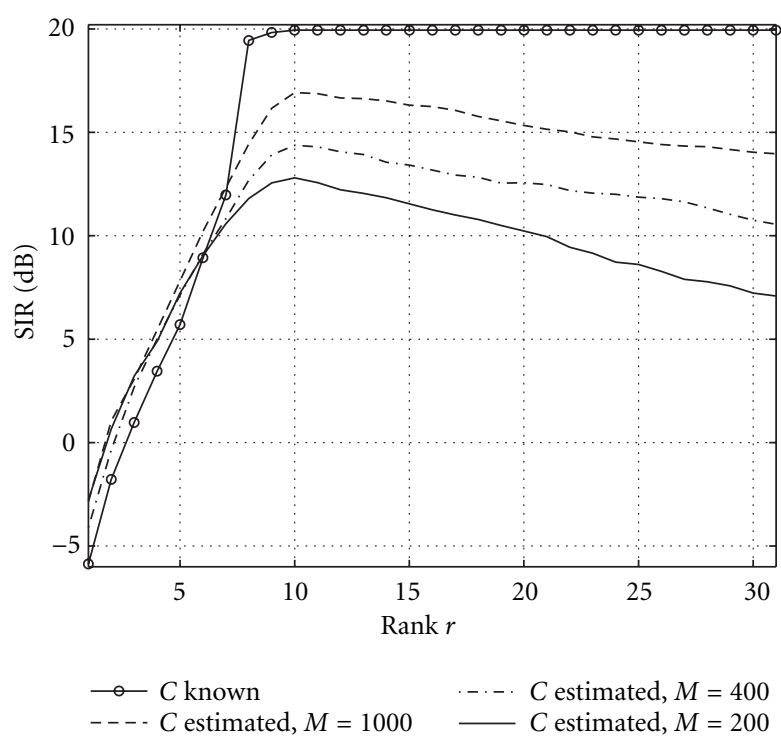

FIGURE 4: SIR of MMSE detectors. $N=31, K=10, \mathrm{SNR}=20 \mathrm{~dB}$, $(K-1)$ interferences' SNRs $20 \mathrm{~dB}$.

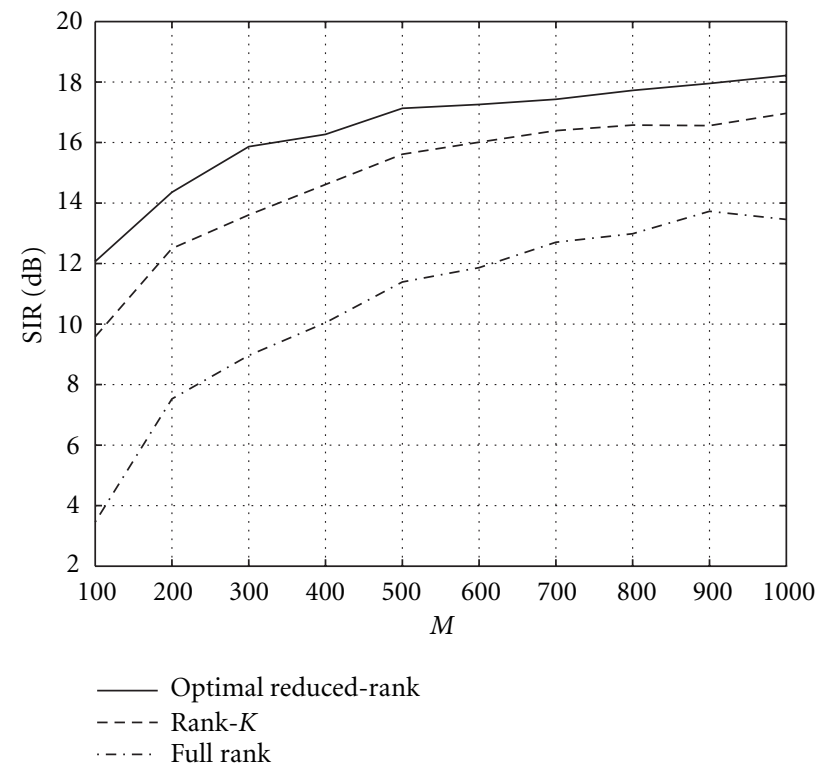

FIGURE 5: SIR of MMSE detectors. $N=31, K=10$, SNR $=20 \mathrm{~dB}$, $(K-1)$ interferences' SNRs are $10 \mathrm{~dB}, 10 \mathrm{~dB}, 10 \mathrm{~dB}, 10 \mathrm{~dB}, 10 \mathrm{~dB}$, $20 \mathrm{~dB}, 30 \mathrm{~dB}, 30 \mathrm{~dB}, 40 \mathrm{~dB}$.

the rank- $K$ MMSE detector, which implies that the optimal reduced-rank MMSE detector has much faster convergence speed. On the other hand, given an $M$, the optimal reducedrank MMSE detector has higher SIR than both rank- $K$ and full-rank MMSE detectors. This demonstrates that, given an effective window size to estimate the covariance matrix, the optimal reduced-rank MMSE detector has the best steadystate performance. 


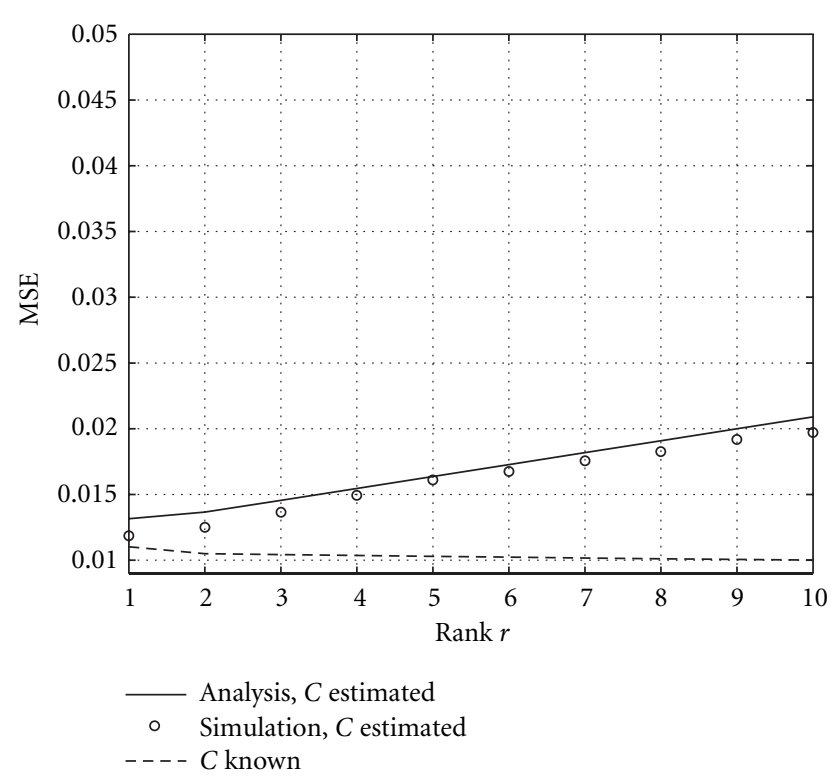

FIgURE 6: MSE of MMSE detectors. $N=31, K=10$, SNR $=20 \mathrm{~dB}$. $(K-1)$ interference users' SNRs are $8.4 \mathrm{~dB}, 8.8 \mathrm{~dB}, 9.2 \mathrm{~dB}, 9.6 \mathrm{~dB}$, $10 \mathrm{~dB}, 10.4 \mathrm{~dB}, 10.8 \mathrm{~dB}, 11.2 \mathrm{~dB}, 11.6 \mathrm{~dB}$.

Example 3 (analytical results). In this example, we compare simulation results with analytical results. There are $K=10$ users in the system. SNRs of different users are chosen to be different so that the ten largest eigenvalues of the covariance matrix are different, which is necessary in the analysis. The number of samples used to estimate the covariance matrix is $M=1000$, and simulation results are obtained by averaging over 2000 independent runs. In Figure 6, nine interfering users have low power. Both simulation and analytical results show that a rank-1 blind MMSE detector has the smallest MSE; whereas, when the covariance matrix is known, rank$K$ MMSE detector has the best performance. In Figure 7, there are both weak and strong interfering users, and a rank2 blind MMSE detector has the best performance. This shows the advantage of the optimal reduced-rank MMSE detector. In Figure 8, interfering users' powers are close to that of the desired user. The signature of the desired user is in the whole signal space. Therefore, the rank- $K$ blind MMSE detector has the smallest MSE. In any case, we can always choose a rank- $r$ MMSE detector with $0<r \leq K$ to achieve the best performance.

Example 4 (simulation of the adaptive reduced-rank MMSE detector). In this example, we use the adaptive algorithm developed in Section 4 to simulate the performance of the adaptive reduced-rank MMSE detector in a dynamic multiple-access channel. The simulation results are depicted in Figure 9. Processing gain is $N=15$ and forgetting factor is $\beta=0.995$. At $t=0$, there are $K=10$ users in the system; among nine interfering users, there are six $10-\mathrm{dB}$ users, one $20-\mathrm{dB}$ user, one 30-dB user, and one $40-\mathrm{dB}$ user. At $t=2000$,

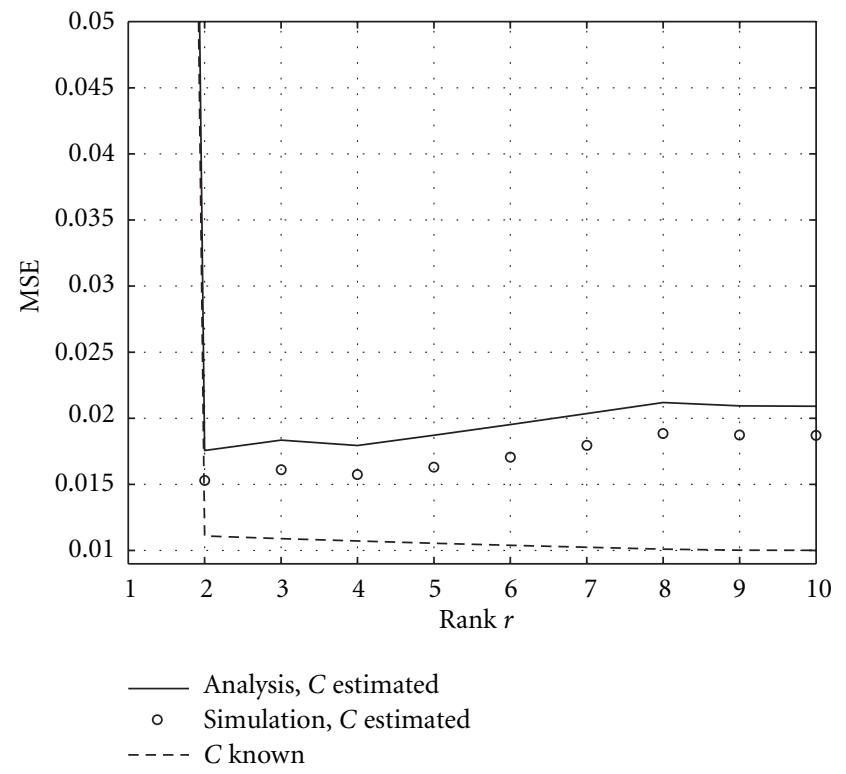

Figure 7: MSE of MMSE detectors. $N=31, K=10$, SNR $=20 \mathrm{~dB}$. $(K-1)$ interference users' SNRs are $8 \mathrm{~dB}, 9 \mathrm{~dB}, 10 \mathrm{~dB}, 11 \mathrm{~dB}, 12 \mathrm{~dB}$, $20 \mathrm{~dB}, 30 \mathrm{~dB}, 31 \mathrm{~dB}, 40 \mathrm{~dB}$.

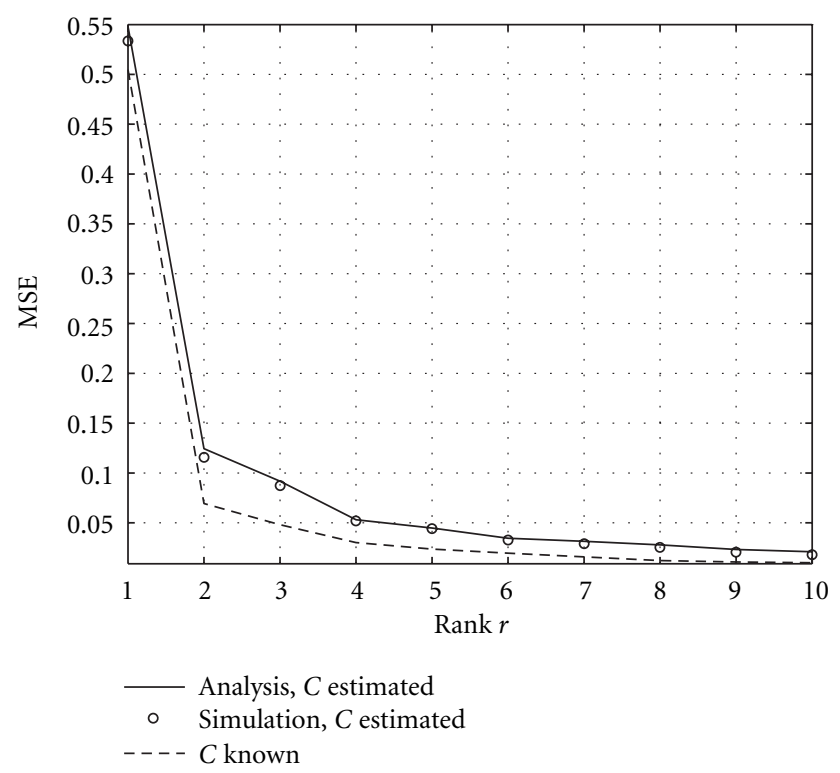

FIgURE 8: MSE of the MMSE detectors. $N=31, K=10$, SNR $=$ $20 \mathrm{~dB},(K-1)$ interference users' SNRs are $16 \mathrm{~dB}, 17 \mathrm{~dB}, 18 \mathrm{~dB}$, $19 \mathrm{~dB}, 20 \mathrm{~dB}, 21 \mathrm{~dB}, 22 \mathrm{~dB}, 23 \mathrm{~dB}, 24 \mathrm{~dB}$.

a $40-\mathrm{dB}$ user enters the channel; at $t=4000$, two $40-\mathrm{dB}$ users exit the channel. The optimal reduced-rank MMSE detector is simulated after first 50 iterations by using ten most recently decided bits to estimate the mean square error. We see that the optimal reduced-rank MMSE detector has approximate $2 \mathrm{~dB}$ advantage over the rank- $K$ MMSE detector, the rank- $K$ 


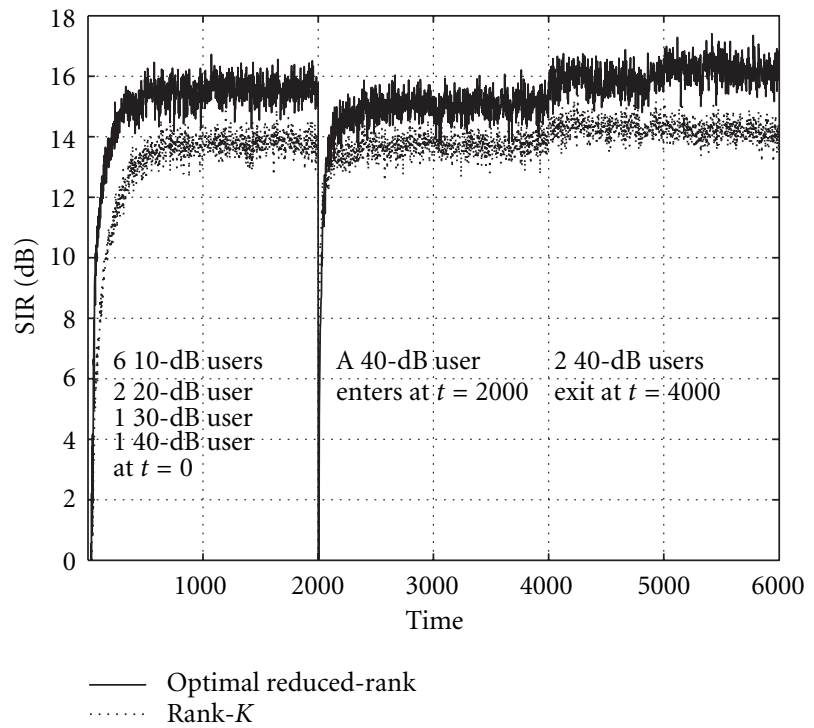

(a) Comparison between the optimal reduced-rank and rank- $K$ MMSE detectors.

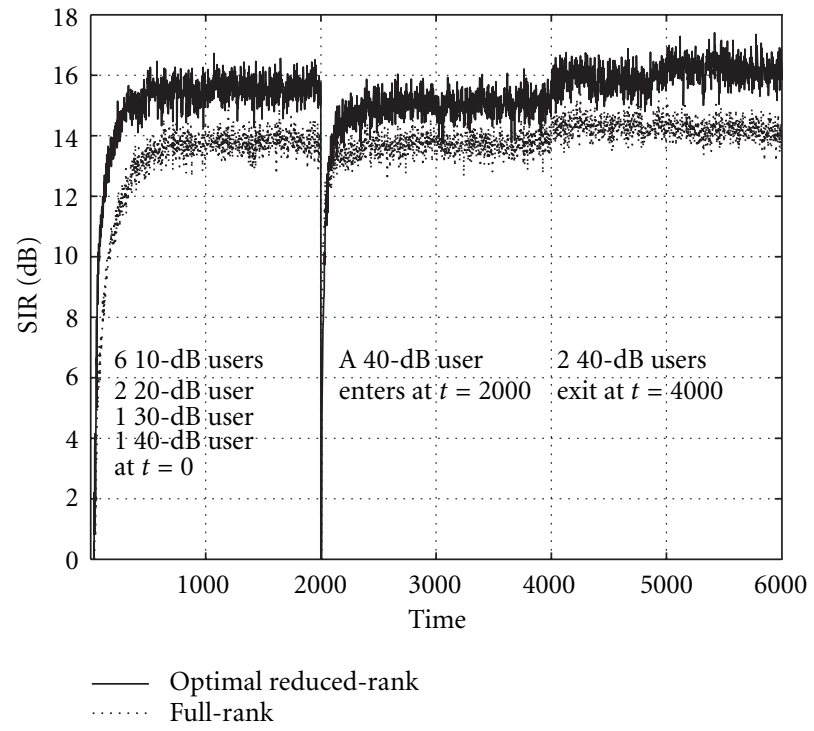

(b) Comparison between the optimal reduced-rank and full-rank MMSE detectors.

FIGURE 9: Performance of the adaptive reduced-rank MMSE detector in a dynamic multiple-access.

MMSE detector has slightly higher SIR than the full-rank MMSE detector in steady-state, and the adaptive algorithm has a fast convergence speed when users enter or exit the system.

\section{CONCLUSIONS}

We developed a blind reduced-rank MMSE detector for DSCDMA by using an estimated covariance matrix of the received signal and forcing the linear MMSE detector to lie in a subspace of the signal space. Our analysis and simulations show that there exists an optimal blind reduced-rank MMSE detector that achieves the highest SIR among all MMSE detectors with different ranks. By properly selecting a subspace, we guarantee that the optimal blind reduced-rank MMSE detector is obtained adaptively. The adaptive blind reducedrank MMSE detector exhibits superior steady-state performance and fast convergence speed in comparison with its full-rank counterpart.

\section{APPENDIX}

\section{DERIVATION OF $\mathbf{H}_{i j}$ AND $F_{i i}$}

Denote the last two terms in (21) as $T_{1}=\hat{\mathbf{c}}_{r}^{T} \mathbf{C} \hat{\mathbf{c}}_{r}$ and $T_{2}=$ $2 \hat{\mathbf{c}}_{r}^{T} \mathbf{p}$. We first derive the first and second partial derivatives of $T_{1}$. The gradient of $T_{1}$ along $\hat{\mathbf{u}}_{i}$ is given by

$$
\begin{aligned}
\mathbf{g}_{1 i} & =\frac{\partial T_{1}}{\partial \hat{\mathbf{u}}_{i}}=2 \frac{\partial \hat{\mathbf{c}}_{r}^{T}}{\partial \hat{\mathbf{u}}_{i}} \mathbf{C} \hat{\mathbf{c}}_{r} \\
& =\frac{2}{\hat{\lambda}_{i}}\left(\mathbf{p} \hat{\mathbf{u}}_{i}^{T}+\mathbf{p}^{T} \hat{\mathbf{u}}_{i} \mathbf{I}_{N}\right) \mathbf{C} \hat{\mathbf{c}}_{r} .
\end{aligned}
$$

If we define $\mathbf{z}_{i}=\left(2 / \hat{\lambda}_{i}\right) \mathbf{C} \hat{\mathbf{c}}_{r}$ and $\mathbf{X}_{i}=\hat{\mathbf{u}}_{i} \mathbf{p}^{T}+\mathbf{p}^{T} \hat{\mathbf{u}}_{i} \mathbf{I}_{N}$, then $\mathbf{g}_{1 i}$ can be expressed as $\mathbf{g}_{1 i}=\mathbf{X}_{i}^{T} \mathbf{z}_{i}$. Since we have

$$
\frac{\partial \mathbf{z}_{i}^{T}}{\partial \hat{\mathbf{u}}_{j}}=\frac{2}{\hat{\lambda}_{i} \hat{\lambda}_{j}} \mathbf{X}_{j}^{T} \mathbf{C}
$$

the Hessian matrix of $T_{1}, \mathbf{H}_{1 j i}$, is given by

$$
\begin{aligned}
\mathbf{H}_{1 j i} & =\left.\frac{\partial \mathbf{g}_{1 i}^{T}}{\partial \hat{\mathbf{u}}_{j}}\right|_{P}=\left.\frac{\partial \mathbf{z}_{i}^{T}}{\partial \hat{\mathbf{u}}_{j}} \mathbf{X}_{i}\right|_{P}=\frac{2}{\lambda_{i} \lambda_{j}} \mathbf{X}_{j}^{T} \mathbf{C} \mathbf{X}_{i} \quad i \neq j, \\
\mathbf{H}_{1 i i} & =\left.\frac{\partial \mathbf{g}_{1 i}^{T}}{\partial \hat{\mathbf{u}}_{i}}\right|_{P}=\left.\frac{\partial\left(\mathbf{z}_{i}^{T} \mathbf{X}_{i}\right)}{\partial \hat{\mathbf{u}}_{i}}\right|_{P} \\
& =\left.\left(\frac{\partial \mathbf{z}_{i}^{T}}{\partial \hat{\mathbf{u}}_{i}} \mathbf{X}_{i}+\left[\frac{\partial \mathbf{x}_{i 1}^{T}}{\partial \hat{\mathbf{u}}_{i}} \mathbf{z}_{i}, \ldots, \frac{\partial \mathbf{x}_{i N}^{T}}{\partial \hat{\mathbf{u}}_{i}} \mathbf{z}_{i}\right]\right)\right|_{P} \\
& =\left.\left(\frac{2}{\hat{\lambda}_{i}^{2}} \mathbf{X}_{i}^{T} \mathbf{C} \mathbf{X}_{i}+\left[\mathbf{Y}_{1} \mathbf{z}_{i}, \ldots, \mathbf{Y}_{N} \mathbf{z}_{i}\right]\right)\right|_{P} \\
& =\frac{2}{\lambda_{i}^{2}} \mathbf{X}_{i}^{T} \mathbf{C} \mathbf{X}_{i}+\frac{2}{\lambda_{i}}\left[\mathbf{Y}_{1} \mathbf{C} \mathbf{c}_{r}, \ldots, \mathbf{Y}_{N} \mathbf{C} \mathbf{c}_{r}\right],
\end{aligned}
$$

where $\left\{\mathbf{x}_{i j}, j=1, \ldots, N\right\}$ are columns of matrix $\mathbf{X}_{i}$ and matrices $\left\{\mathbf{Y}_{j}, j=1, \ldots, N\right\}$ are defined as

$$
\mathbf{Y}_{j}=\mathbf{P}_{j}+\mathbf{p}(j) \mathbf{I}_{N}
$$

with $\mathbf{P}_{j}$ being a matrix whose $j$ th column is vector $\mathbf{p}$ and other columns being zero vectors. The term $T_{1}$ can also be expressed as 


$$
\begin{aligned}
T_{1}= & \mathbf{p}^{T} \sum_{j=1}^{r} \frac{\hat{\mathbf{u}}_{j} \hat{\mathbf{u}}_{j}^{T}}{\hat{\lambda}_{j}} \mathbf{C} \sum_{j=1}^{r} \frac{\hat{\mathbf{u}}_{j} \hat{\mathbf{u}}_{j}^{T}}{\hat{\lambda}_{j}} \mathbf{p} \\
= & \mathbf{p}^{T}\left(\frac{\hat{\mathbf{u}}_{i} \hat{\mathbf{u}}_{i}^{T}}{\hat{\lambda}_{i}}+\sum_{\substack{j=1 \\
j \neq i}}^{r} \frac{\hat{\mathbf{u}}_{j} \hat{\mathbf{u}}_{j}^{T}}{\hat{\lambda}_{j}}\right) \mathbf{C}\left(\frac{\hat{\mathbf{u}}_{i} \hat{\mathbf{u}}_{i}^{T}}{\hat{\lambda}_{i}}+\sum_{\substack{j=1 \\
j \neq i}}^{r} \frac{\hat{\mathbf{u}}_{j} \hat{\mathbf{u}}_{j}^{T}}{\hat{\lambda}_{j}}\right) \mathbf{p} \\
= & \frac{1}{\hat{\lambda}_{i}^{2}} \mathbf{p}^{T} \hat{\mathbf{u}}_{i} \hat{\mathbf{u}}_{i}^{T} \mathbf{C} \hat{\mathbf{u}}_{i} \hat{\mathbf{u}}_{i}^{T} \mathbf{p}+\frac{2}{\hat{\lambda}_{i}} \mathbf{p}^{T} \hat{\mathbf{u}}_{i} \hat{\mathbf{u}}_{i}^{T} \mathbf{C} \sum_{\substack{j=1 \\
j \neq i}}^{r} \frac{\hat{\mathbf{u}}_{j} \hat{\mathbf{u}}_{j}^{T}}{\hat{\lambda}_{j}} \mathbf{p} \\
& +\mathbf{p}^{T} \sum_{\substack{j=1 \\
j \neq i}}^{r} \frac{\hat{\mathbf{u}}_{j} \hat{\mathbf{u}}_{j}^{T}}{\hat{\lambda}_{j}} \mathbf{C} \sum_{\substack{j=1 \\
j \neq i}}^{r} \frac{\hat{\mathbf{u}}_{j} \hat{\mathbf{u}}_{j}^{T}}{\hat{\lambda}_{j}} \mathbf{p} .
\end{aligned}
$$

From (A.5), the first partial derivative of $T_{1}$ with respect to $\hat{\lambda}_{i}$ is given by

$$
\frac{\partial T_{1}}{\partial \hat{\lambda}_{i}}=-\frac{2}{\hat{\lambda}_{i}^{3}} \mathbf{p}^{T} \hat{\mathbf{u}}_{i} \hat{\mathbf{u}}_{i}^{T} \mathbf{C} \hat{\mathbf{u}}_{i} \hat{\mathbf{u}}_{i}^{T} \mathbf{p}-\frac{2}{\hat{\lambda}_{i}^{2}} \mathbf{p}^{T} \hat{\mathbf{u}}_{i} \hat{\mathbf{u}}_{i}^{T} \mathbf{C} \sum_{\substack{j=1 \\ j \neq i}}^{r} \frac{\hat{\mathbf{u}}_{j} \hat{\mathbf{u}}_{j}^{T}}{\hat{\lambda}_{j}} \mathbf{p}, \text { (A.6) }
$$

and then, the second partial derivative of $T_{1}$ with respect to $\hat{\lambda}_{i}$ is found as

$$
\left.\frac{\partial^{2} T_{1}}{\partial \hat{\lambda}_{i}^{2}}\right|_{P}=\frac{6}{\lambda_{i}^{4}} \mathbf{p}^{T} \mathbf{u}_{i} \mathbf{u}_{i}^{T} \mathbf{C} \mathbf{u}_{i} \mathbf{u}_{i}^{T} \mathbf{p}+\frac{4}{\lambda_{i}^{3}} \mathbf{p}^{T} \mathbf{u}_{i} \mathbf{u}_{i}^{T} \mathbf{C} \sum_{\substack{j=1 \\ j \neq i}}^{r} \frac{\mathbf{u}_{j} \mathbf{u}_{j}^{T}}{\lambda_{j}} \mathbf{p} .
$$

It is easily shown that the second term in (A.7) is equal to zero and $\mathbf{u}_{i}^{T} \mathbf{C u}_{i}=\lambda_{i}$. Thus, we have

$$
\left.\frac{\partial^{2} T_{1}}{\partial \hat{\lambda}_{i}^{2}}\right|_{P}=\frac{6}{\lambda_{i}^{3}} \mathbf{p}^{T} \mathbf{u}_{i} \mathbf{u}_{i}^{T} \mathbf{p} .
$$

Next we determine the derivatives of $T_{2}$ that can also be expressed as

$$
T_{2}=2 \sum_{i=1}^{r} \frac{\left(\mathbf{p}^{T} \hat{\mathbf{u}}_{i}\right)^{2}}{\hat{\lambda}_{i}} .
$$

Gradient of $T_{2}$ along $\hat{\mathbf{u}}_{i}$ is given by

$$
\mathbf{g}_{2 i}=\frac{\partial T_{2}}{\partial \hat{\mathbf{u}}_{i}}=\frac{4}{\hat{\lambda}_{i}} \mathbf{p}^{T} \hat{\mathbf{u}}_{i} \mathbf{p}
$$

From (A.12), we have $\mathbf{H}_{2 i j}=\partial \mathbf{g}_{2 i}^{T} / \partial \hat{\mathbf{u}}_{j}=\mathbf{0}$ for $i \neq j$, and $\mathbf{H}_{2 i i}$ is found as

$$
\mathbf{H}_{2 i i}=\left.\frac{\partial \mathbf{g}_{2 i}^{T}}{\partial \hat{\mathbf{u}}_{i}}\right|_{P}=\frac{4}{\lambda_{i}} \mathbf{W}
$$

where the ith column of matrix $\mathbf{W}$ is $\mathbf{p}(i) \mathbf{p}$. The second derivative of $T_{2}$ with respect to $\hat{\lambda}_{i}$ at point $P$ is given by

$$
\left.\frac{\partial^{2} T_{2}}{\partial \hat{\lambda}_{i}^{2}}\right|_{P}=\frac{4\left(p^{T} \mathbf{u}_{i}\right)^{2}}{\lambda_{i}^{3}}
$$

Finally, $F_{i i}$ in (24) is given by $F_{i i}=\partial^{2} T_{1} /\left.\partial \hat{\lambda}_{i}^{2}\right|_{P}-\partial^{2} T_{2} /\left.\partial \hat{\lambda}_{i}^{2}\right|_{P}$, and $\mathbf{H}_{i j}$ in (24) is given by $\mathbf{H}_{i j}=\mathbf{H}_{1 i j}-\mathbf{H}_{2 i j}$.

\section{ACKNOWLEDGMENT}

This work was presented in part at the 33rd Asilomar Conference on Signals, Systems, and Computer, Pacific Grove, Calif, October 1999.

\section{REFERENCES}

[1] S. Verdú, Multiuser Detection, Cambridge University Press, Cambridge, UK, 1998.

[2] R. Lupas and S. Verdú, "Linear multiuser detectors for synchronous code-division multiple-access channels," IEEE Transactions on Information Theory, vol. 35, no. 1, pp. 123136, 1989.

[3] U. Madhow and M. Honig, "MMSE interference suppression for direct-sequence spread spectrum CDMA," IEEE Trans. Communications, vol. 42, no. 12, pp. 3178-3188, 1994.

[4] Z. Xie, R. T. Short, and C. K. Rushforth, "A family of suboptimum detectors for coherent multiuser communications," IEEE Journal on Selected Areas in Communications, vol. 8, no. 4, pp. 683-690, 1990.

[5] M. Honig, U. Madhow, and S. Verdú, "Blind adaptive multiuser detection," IEEE Transactions on Information Theory, vol. 41, no. 4, pp. 944-960, 1995.

[6] X. Wang and H. V. Poor, "Blind multiuser detection: A subspace approach," IEEE Transactions on Information Theory, vol. 44, no. 2, pp. 677-690, 1998.

[7] B. Yang, "Projection approximation subspace tracking," IEEE Trans. Signal Processing, vol. 44, no. 1, pp. 95-107, 1995.

[8] Y. Song and S. Roy, "Blind adaptive reduced-rank detection for DS-CDMA signals in multipath channels," IEEE Journal on Selected Areas in Communications, vol. 17, no. 11, pp. 19601970, 1999.

[9] X. Wang and H. V. Poor, "Blind equalization and multiuser detection in dispersive CDMA channels," IEEE Trans. Communications, vol. 46, no. 1, pp. 91-103, 1998.

[10] X. Cai, H. Ge, and A. N. Akansu, "Low-rank MMSE detector for synchronous DS-CDMA," in Proc. 33rd Asilomar Conference on Signals, Systems and Computer, pp. 940-944, Pacific Grove, Calif, USA, October 1999.

[11] J. S. Goldstein, I. S. Reed, and L. L. Scharf, "A multistage representation of the Wiener filter based on orthogonal projections," IEEE Transactions on Information Theory, vol. 44, no. 7, pp. 2943-2959, 1998.

[12] M. Honig and W. Xiao, "Performance of reduced-rank linear interference suppression," IEEE Transactions on Information Theory, vol. 47, no. 5, pp. 1928-1946, 2001.

[13] J. S. Goldstein and I. S. Reed, "Subspace selection for partially adaptive sensor array processing," IEEE Trans. on Aerospace and Electronics Systems, vol. 33, no. 2, pp. 539-543, 1997.

[14] X. Cai, H. Ge, and A. N. Akansu, "Low-rank minimum variance CDMA receiver in multipath channels," in Proc. IEEE Int. Conf. Acoustics, Speech, Signal Processing, pp. 2877-2880, Istanbul, Turkey, June 2000.

[15] M. Wax and T. Kailath, "Detection of signals by information theoretic criteria," IEEE Trans. Acoustics, Speech, and Signal Processing, vol. 33, no. 2, pp. 387-392, 1985.

[16] D. R. Brillinger, Time Series: Data Analysis and Theory, Holt, Rinehart and Winston, New York, NY, USA, 1975.

[17] R. P. Gupta, "Asymptotic theory for principal component analysis in the complex case," J. Indian Statist. Assoc., vol. 3, pp. 97-106, 1965. 
[18] G. H. Golub and C. F. V. Loan, Matrix Computations, The Johns Hopkins University Press, Baltimore, Md, USA, 1996.

[19] E. M. Dowling, L. P. Ammann, and R. D. DeGroat, "A TQRiteration based adaptive SVD for real time angle and frequency tracking," IEEE Trans. Signal Processing, vol. 42, no. 4, pp. 914-926, 1994.

[20] P. Strobach, "Low-rank adaptive filters," IEEE Trans. Signal Processing, vol. 44, no. 12, pp. 2932-2947, 1995.

[21] B. Yang, "An extension of the PASTd algorithm to both rank and subspace tracking," IEEE Signal Processing Letters, vol. 2, no. 1, pp. 179-182, 1995.

[22] H. V. Poor and S. Verdú, "Probability of error in MMSE multiuser detection," IEEE Transactions on Information Theory, vol. 43, no. 3, pp. 858-871, 1997.

Xiaodong Cai received the B.S. degree from Zhejiang University, China, the M.S. degree from the National University of Singapore, Singapore, and the Ph.D. degree from New Jersey Institute of Technology in December 2000, all in electrical engineering. From February 2001 to June 2001, he was a member of Technical Staff at Lucent Technologies, NJ, working on W-CDMA project. From July 2001 to October 2001, he was a

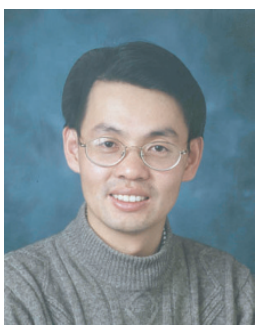
Senior System Engineer at Sony Technology Center in San Diego, Calif, involved in developing high data rate wireless modem. Since November 2001, he has been a Post-Doctoral Research Associate in the department of Electrical and Computer Engineering, University of Minnesota, Minneapolis. His research interests lie in the areas of communication theory, signal processing, and wireless networks.

Hongya Ge received the B.S. degree from the University of Electronic Science and Technology of China (UESTC), Chengdu, China, the M.S. degree from the Nanjing University of Aeronautics and Astronautics, Nanjing, China, and the Ph.D. degree from the University of Rhode Island, Kingston, RI, in 1982, 1985, and 1994, respectively, all in electrical engineering. From 1986 to 1990, Dr. Ge was with the Department of

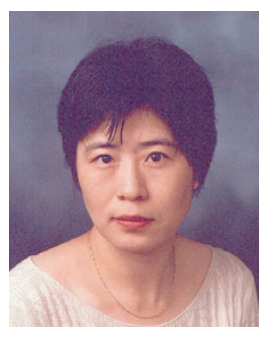
Information and Electronics at the Zhejiang University, as Lecturer/Researcher, teaching and researching in radar signal processing, communication theory, and numerical analysis. Since 1995, she has been with the Department of Electrical and Computer Engineering, New Jersey Institute of Technology, Newark, NJ, where she is currently an Associate Professor. During the academic year 2000-2001, she was with the Applied Research Department of Telcordia Technologies, Inc. NJ, working on broadband wireless access, MIMO transceiver systems, and wireless network security projects. Her research interests are in the general areas of statistical and array signal processing and digital wireless communications.

Ali N. Akansu received the B.S. degree from the Technical University of Istanbul in 1980, and received the M.S. and Ph.D. degrees from the Polytechnic University in 1983 and 1987, respectively, all in electrical engineering. Since 1987, he has been with the New Jersey Institute of Technology, where he is, currently, a Professor of electrical and computer engineering. He was a Cofounder and Director of the New Jersey Center for Multimedia Research between 1996-2000. He was the Vice President for Research and Design
(R \& D) of the IDT Corporation between 2000-2001. He has been the Founding President of PixWave Inc. His industrial affiliations also include his visits to IBM T.J., Watson Research Center and at GEC-Marconi Electronic Systems Corp. during the summers of 1989,1996 , and 1992, respectively. He serves as a Consultant to the industry and sits on the boards of a few Internet start-up companies. He coauthored and coedited three books and many papers on his research. His current research is on signal and transform theories with applications in multimedia and communications. 